\title{
Primäre lymphatische Organe (Km, Thymus und peripheres Blut)
}

Christine Greil (Klinikerin), Anna Verena Frey, Maximilian Seidl (Pathologen) unter Mitarbeit von: Thomas Cerny, Kirill Karlin

18.1 Die Sicht des Klinikers - 124

18.2 Die Sicht des Pathologen - 124

18.3 Knowledge-Bites -125

18.4 PathoMap Knochenmark und Thymus - 126

18.5 Vertiefungsseite: Peripheres Blut -128 


\subsection{Die Sicht des Klinikers}

\section{Allgemeines zur Hämatologie}

Anamnese u. Leitsymptome:

- Akutes (AML, ALL, aggressive Lymphome) vs. chronisches (MPN, MDS, niedrig maligne Lymphome zB CLL) Geschehen?

- Anzeichen hämatopoetischer Insuffizienz? B-Symptomatik (Fieber, Nachtschweiss, Gewichtsverlust)? Oberbauchbeschwerden? Vergrösserte Lymphknoten? (• Abb. 3)

- PA: Noxen, Medikamente? St.n. Radio-/Chemotherapie?

- Familien- u. Berufsanamnese (zB Benzol-Exposition?).

Diagnostik:

- Klinische Untersuchung: - Abb. 3.

- Labor: Differentialblutbild, Gerinnungsstatus, LDH (Zellumsatzmarker), Haptoglobin u. ggf. Immunhämatologie (Hämolyse?), Folsäure/Vit. B12, Virusserologien.

- Knochenmarkpunktion: zunächst Aspiration $\rightarrow$ Gewinnung von Zellen für Zytologie („Ausstrich“), Immunzytologie (FACS), Zytogenetik (FISH), Molekulargenetik; danach Stanzbiopsie $\rightarrow$ Gewebeprobe für Histologie.

- Lymphknotenextirpation (falls nicht möglich: -biopsie).

- Bildgebung: Sonographie, ggf. CT/MRT, selten PET-CT.

- Liquorpunktion: b. ALL immer, b. AML/Lymphomen nur b. Vda Meningeosis.

Therapie

- IdR Systemerkrankung - Lokaltherapie nicht zielführend.

- Benigne Ursachen ( $z B$ Vitaminmangel, myelotox. Medikamente): Substratsubstitution, Noxen meiden etc.

- Maligne: supportiv (Wachstumsfaktoren, Transfusion, Infekttherapie/-prophylaxe) u. antineoplastisch (Zytoreduktiva, $\mathrm{Cx}$-/Rx-Therapie, Immunologika, ggf. HSCT).

\section{Fokus: Der Patient mit akuter Leukämie}

- Anamnestisch meist kurzer Verlauf, berichtet Leistungsabfall, Gliederschmerzen u. B-Symptome (• Abb. 3).

- Gesunde Hämatopoese wird meist vollständig verdrängt, daher Infekte (Mucositis, Abszesse) u. Blutungsneigung (hauptsächlich Petechien aufgr. Tz-Penie).

- Befall anderer Organe: Lymphadenopathie, Gingivahyperplasie, Chlorome (extramedullärer myeloider Tumor, ua kutan, auch abdominell, ossär, zerebral), Hepatosplenomegalie.

- Bei Meningeosis: Kopf-Sz, Nausea/Emesis, Sehstörungen.

- Selten Leukostase b. extrem hohen Leukozytenzahlen: pneumonische Infiltrate mit Dyspnoe, zerebrale Ischämien/Hämorrhagien, arterielle Verschlüsse.

\subsection{Die Sicht des Pathologen}

Ausgangslage: die angeborene/unreife Abwehr entsteht

- Das Knochenmark ist Ausgangspunkt aller Blutzellen. Der damit verbundene hohe Zellumsatz ist der Boden für die Entstehung von Neoplasien.

- Die Veränderungen der Hämatopoese sind grob unterteilbar in primäre Blutbildungsstörungen (Aktion) vs. sekundäre Blutbildungsstörungen (Reaktion).

- Der Thymus ist der Reifungsort der T-Zellen. Veränderungen umfassen primäre o. sekundäre Tumoren (Thymom, Thymuskarzinom, vs. Lymphom, Metastasen), Entzündungen (Myasthenia Gravis) o. Hypotrophie/Aplasie iF angeborener Immundefekte (DiGeorge Syndrom).

- Der Thymus durchläuft nach Säuglings-/Kindesalter eine Altersinvolution (fettgewebig Umbildung u. Atrophie) Repertoire an T-Zellen kann nicht weiter zunehmen.

\section{Diagnostik}

- Der Pathologe erhält Knochenmarkstrepanat/-ausstriche, mediastinoskopische Biopsien o. Thymusresektate.

- Klinische Angaben (Symptomdauer, Noxen u. Medikamente, Blutwerte) sind für eine korrekte Diagnose unerlässlich.

- Ebenso entscheidend: hochwertige präanalytische Qualität (ausreichende Probengrösse, schonende Entkalkung, Vermeidung von Quetschartefakten durch Instrumente).

- Histologisch können MPN, AML, ALL, CLL u. Lymphome gut am KM-Trepanat diagnostiziert werden. Molekulargenetik zunehmend wichtig für deren Subklassifizierung.

- Für die Diagnose MDS bedarf es der Korrelation von Aspirat u. Molekularzytogenetik.

- B. akuten Leukämien u. MDS ist aktuell der molekulargenetische Subtyp für den Therapieplan wichtig. Hierfür sind Immunhistochemie, Zytogenetik u. Molekularpathologie notwendig.

- Thymom: histologische Abgrenzung zu Thymus-CA anhand Wachstumsmuster u. Ausdehnung wichtig.

\section{Besonderheit: reaktive Knochenmarksveränderungen}

- Substratmangel, Regenerationsphasen, Infekte, Noxen, Medikamente o. verdrängende Prozesse können eine primäre Neoplasie des Knochenmarks imitieren.

- Diese „Mimics“ durch reaktive Veränderungen können schnell zu Fehldiagnosen führen (zB Folsäuremangel = initiales MDS). Die richtige Diagnose kann oft erst im Verlauf (zB nach Substratsubstitution) mittels Rebiopsie unter Mitberücksichtigung der klinischen Dynamik gestellt werden.

Schwierige Stellen

Hämatologie ist ein Zusammenspiel von Kompartimenten, die nicht isoliert voneinander funktionieren. Aus didaktischer Sicht ist eine Trennung zunächst sinnvoll, um die Grundfunktionen eines jeden Kompartiments zu begreifen $\left(1^{\circ}\right.$ lymphatisch $=$ Bildung, intravasal $=$ Interaktion/Transport, $2^{\circ}$ lymphatisch $=$ Aktivierung $/$ Ausreifung $\left./ \mathrm{Abbau}\right)$. In einem zweiten Schritt sollte dann aber die gegenseitige Abhängigkeit erlernt werWden, da im Klinikalltag eine Trennung oft nicht sinnvoll möglich ist (leukämischer Verlauf eines Lymphoms mit Befall des Knochenmarks, Lymphadenopathie b. Leukämie, sekundäre Splenomegalie b. Knochenmarkinsuffizienz). Eine Herausforderung kann demgegenüber zB die Tatsache sein, dass eine krankhafte Hämatopoese nicht zwangsläufig auffällige periphere Blutwerte zur Folge haben muss. Manchmal kann das Zusammenspiel der Kompartimente auch über ein Problem hinwegtäuschen. 

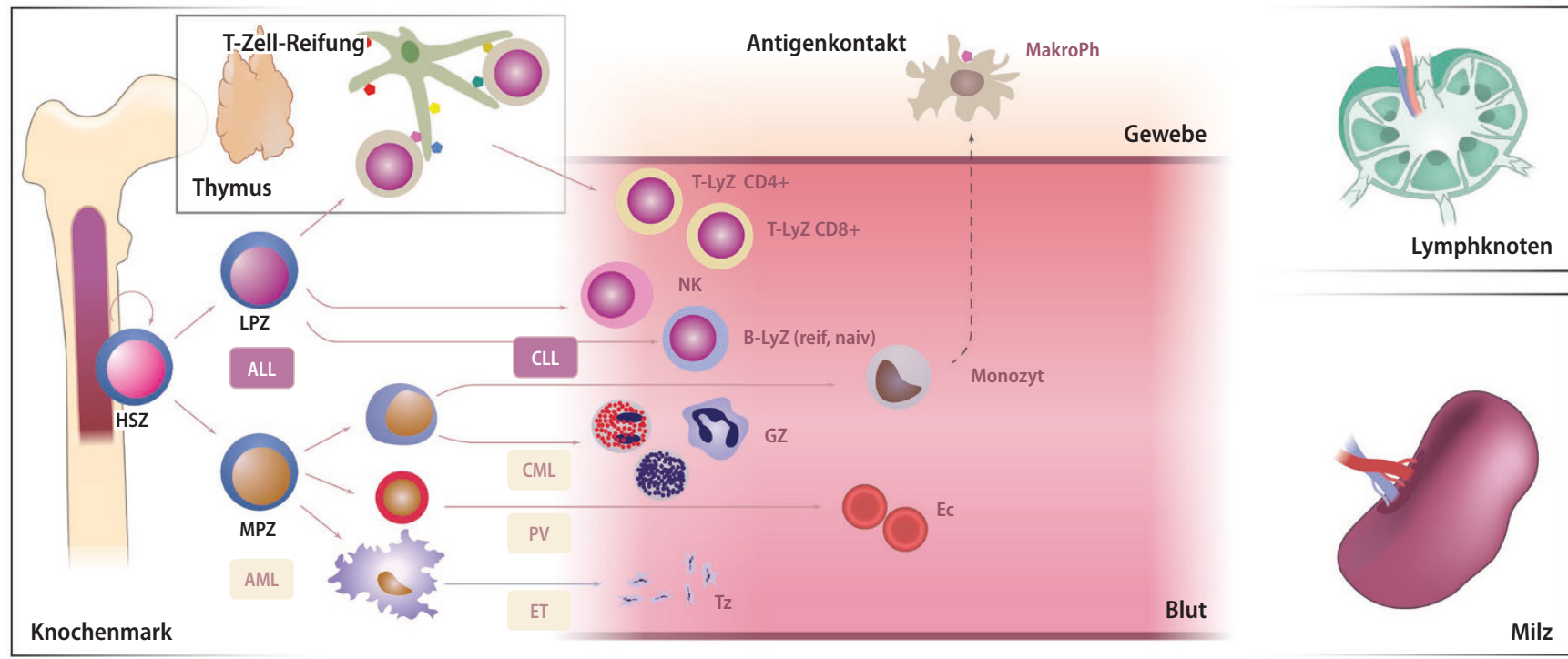

- Abb. 1 Vereinfachte Darstellung d. Hämatopoese, welche beim Erw. im Knochenmark stattfindet. Aus der multipotenten Hämatopoetischen Stammzelle (HSZ) entstehen zwei Hauptlinien: myeloische Reihe aus d. Myeloischen Progenitorzelle (MPZ) u. lymphatische Reihe aus der Lymphatischen Progenitorzelle (LPZ). Dazu dargestellt sind die assoziierten primären Neoplasien. (๑Cerny, Karlin, 2018 [18.1])
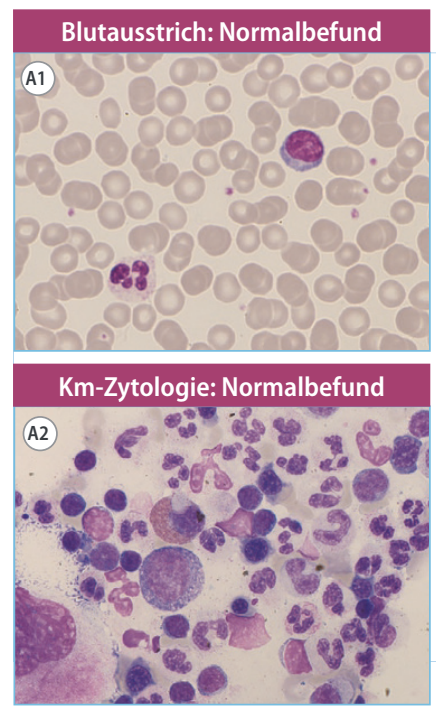
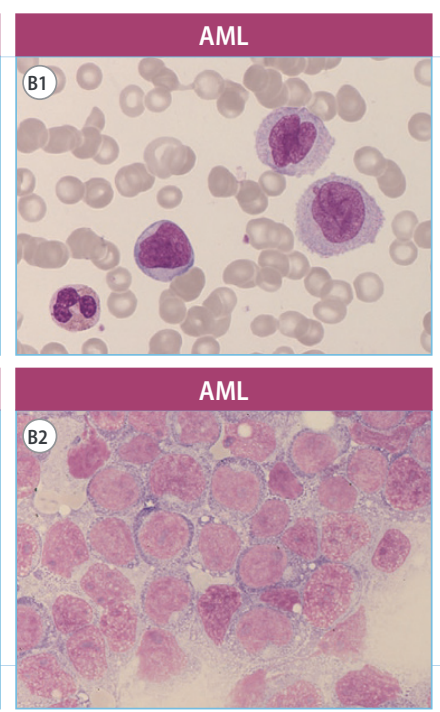
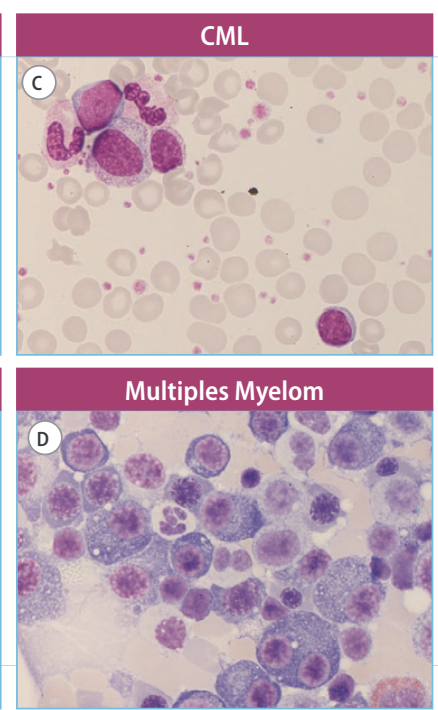
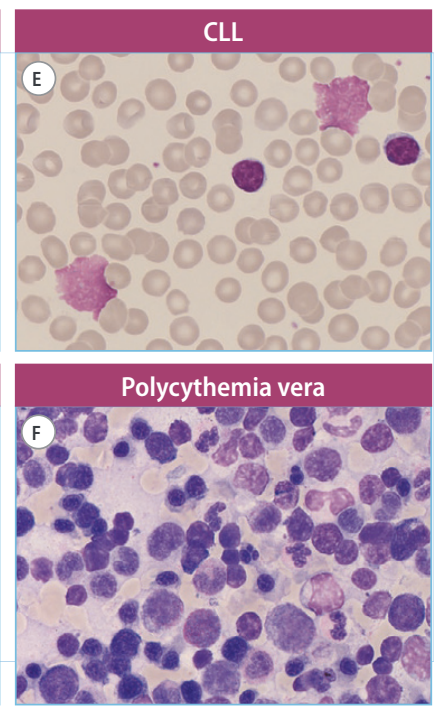

- Abb. 2 Peripherer Blutausstrich u. Knochenmark-Aspirationszytologie b. hämatologischen Neoplasien. A) Normalbefund. B1) Grosse Blasten zT mit Nukleoli, Hiatus leucaemicus. B2) Monomorphe Blasteninfiltration. C) Linksverschiebung bis zum Blasten, ohne Hiatus leucaemicus. D) Deutlich erhöhter Plasmazellnachweis, zT atypische (doppelkernige) Formen. E) Lymphozytose, Gumprecht'sche Kernschatten. F) Zellularität erhöht, Erythropoese dominant.

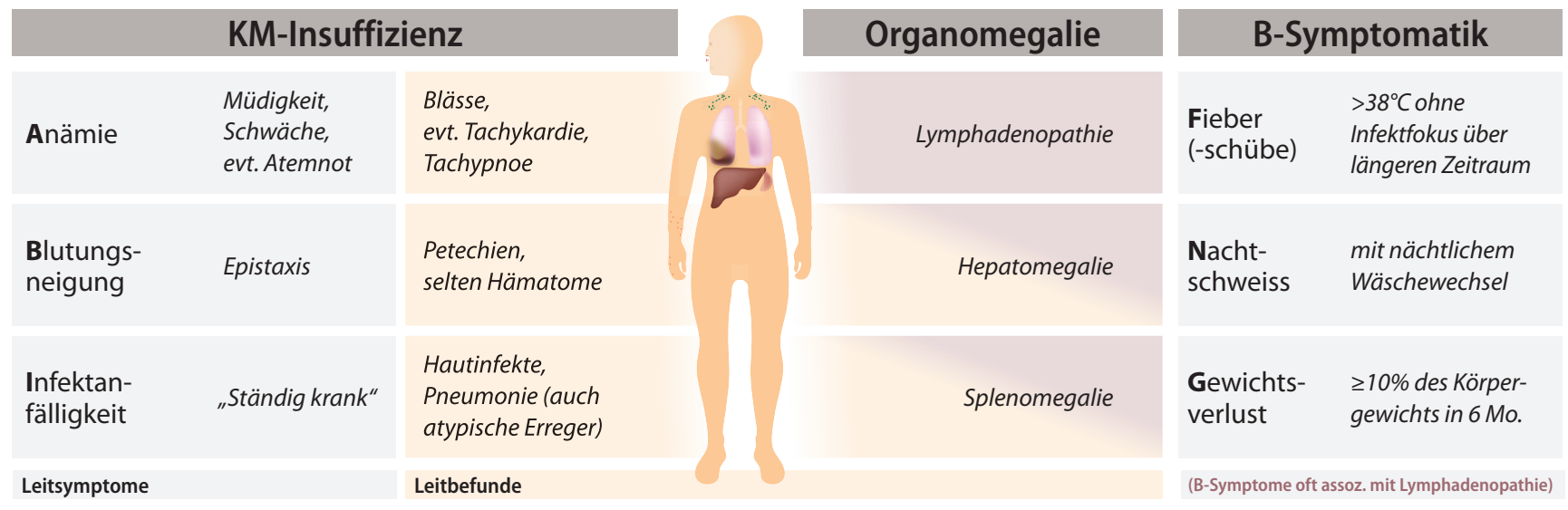

- Abb. 3 Wichtigste hämatologische Leitsymptome u. Leitbefunde in der Übersicht. Die farbige Hinterlegung verweist auf die gestörte Zellreihe: myeloische Zellreihe (orange), lymphatische Zellreihe (violett). 
Knochenmark

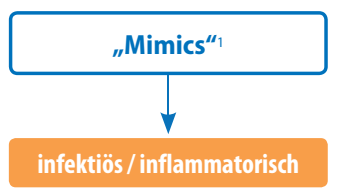

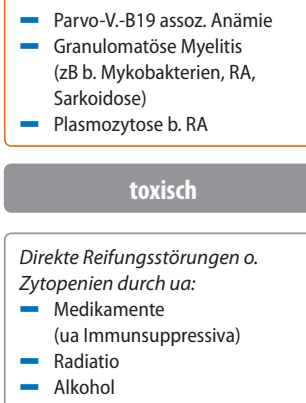

\begin{tabular}{|l|}
\multicolumn{1}{|c|}{ metabolisch } \\
\hline - Renale Anämie b. EPO-Mangel \\
iF CNI \\
- Mikrozytäre Anämie b. Eisen- \\
Mangel \\
- Sekundäre Polyglobulie b. \\
hypoxischen Zuständen
\end{tabular}

\begin{tabular}{|l|}
\hline \multicolumn{1}{|c|}{ autoimmun } \\
\hline - Immunagranulozytose iF Bil- \\
dung von Anti-Granulozyten- \\
Antikörpern (autoimmun u. \\
haptenvermittelt, zB Medika- \\
mente: Metamizol, Clozapin) \\
- PRCA (pure red cell anaemia) \\
ua durch Autoantikörper \\
- Hyperplastische Megakaryo- \\
poese b. ITP
\end{tabular}

\section{neoplastisch}

- Verdrängungsmyelopathie b. zB Metastasenbefall (=Knochenmark-Karzinose)

\section{myeloproliferative Neoplasien}

Polycythemia vera (PV)

E Männer ø60J., selten (1:1 Mio) $\ddot{A}$ in 95\% JAK2-Mutation

$\begin{array}{ll}\text { A } & \text { in 95\% JAK2-Mutation } \\ P & \text { klonale Expansion d. Ec-Poese }\end{array}$

K Plethora, Erythromelalgie, hydrogener Pruritus, Hepatomegalie

Ko Thromben, Myelofibrose

D $\quad H b>16$ (f) resp. 16.5g/dl (m), EPO

DD \ sek. Polycythämie (EPO $\uparrow)$

T Aderlass, ASS, HU, Ruxolitinib

\section{Essentielle Thrombozythämie \\ E $f>m$, bimodal $30 / 60$ J \\ Ä in 60\% JAK2-Mutatation \\ $\mathrm{P} \quad \mathrm{Tz} \uparrow \uparrow$, aber zT Funktionsverlust \\ $\mathrm{K}$ Thromben $\mathrm{u}$. Blutungen \\ D Tz persistierend $>450 \mathrm{G} / \mathrm{L}$ \\ T ASS, Hydroxyurea (HU)}

\section{(Primäre) Myelofibrose (MF)}

E 60-70J.

Ä TGFß ex entarteten MKZ

P Km-Fibrose verdrängt Blutbildung ( $z B$ in Milz/ Leber)

K Mega-Milz, Hepatomegalie

D „Punctio sicca“ b. Km-Punktion

$\rightarrow \mathrm{Km}$-Biopsie notwendig

T HSCT (Fibrosierung reversibel!)

JAK2, MPL o. Calretikulin +/-

\section{Chronische myeloische}

Leukämie (CML) (BCR/ABL+)

E $m>f, 40-60 \mathrm{~J}$.

Ä $\mathrm{t}(9 ; 22) \rightarrow$ bcr-abl-Fusionsgen mit Tyrosinkinaseaktivität

$\mathrm{P} \quad$ Myeloblasten $\uparrow \mathrm{n} / \mathrm{e} / \mathrm{bGZ} \uparrow \uparrow$

K chronische Phase $\rightarrow$ Akzeleration $\rightarrow$ Blastenschub

D $\quad \mathrm{Lc} \uparrow \uparrow, \mathrm{bGZ} \uparrow, \mathrm{LAP}(-)^{3}$

T - Chronisch: Tyrosinkinaseinhibit. - Im Blastenschub: wie AML

\section{Weitere MPN}

- Chronische Neutrophilenleukämie

- Chronische Eosinophilenleukämie

MPN/MDS-Overlapsyndrome

- Chronische myelomonozytäre Leuk.

- Atypische CML (BCR/ABL-)

Aus myeloischer Reihe
Neoplasie ${ }^{2}$

\begin{tabular}{|c|c|c|}
\hline myelodysplastische Syndrome & akute Leukämie & Sonstige \\
\hline $\begin{array}{l}\text { Myelodysplastische } \\
\text { Syndrome (MDS) } \\
\text { E M }>\text { F, } \varnothing 70 J \\
\text { Ä de-novo; RF: Chemikalien (Ben- } \\
\text { zene), Rauchen, positive FA } \\
\text { P } 10 \% \text { Dysplasie in } \geq 1 \text { Zellreihe; }\end{array}$ & $\begin{array}{l}\text { Akute myeloische } \\
\text { Leukämie (AML) } \\
\text { E jedes Alter möglich, häufiger b. } \\
\text { Älteren } \\
\text { A RF: MDS, Cx, Rx, Genetik } \\
\text { (Trisomie 21) ua. }\end{array}$ & 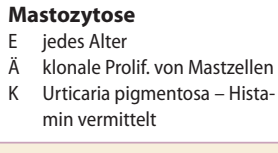 \\
\hline
\end{tabular}

P. $10 \%$ Dysplasie in $\geq 1$ Zelreihe;

unterschiedliche Subtypen

$5 q-D e l e t i o n$ ist per Def. MDS

D Zytopenie peripher, Hepatosplenomegalie (Abbau $\uparrow$ der

fehlerhaften" Zellen)

T abhängig vom Risikoprofil

$\operatorname{Pr}$ Risikogruppen basieent auf

IPSS-R nach: Karyotyp, Blastengehalt, Hb-Wert, Tz-Anzahl, Neutrophilenzahl o. bestimmte zytogenetischen Abnormalitäten

Subtypen $z B$ :

- MDS mit Ringsideroblasten

- MDS mit unilineärer Dysplasie

- MDS mit multilineärer Dysplasie

- MDS mit isolierter del(5q)

- MDS nicht weiter klassifizierbar

Bei Blastenanstieg: Übergang in Leukämie möglich

(Trisomie 21) ua.

P > 20\% Blasten in KM o. Blut

D Histologie, Zytogenetik, Zytologie, Zytochemie, Laborwerte

T Cx-Therapie, ggf. HSCT, je nach Subtyp

Pr je nach Entität

Subtypen zB:

- AML mit Keimbahnmutation (Mutation in CEBPA-Gen)

- AML mit rekurrenten genetischen Abnormalitäten

- AML aus MDS

- AML therapieassoziiert

- AML, nicht weiter klassifizierbar

Spezialfall:

Akute Pro-Myelozyten Leukämie

(APML)

E $5 \%$ der AML, va bei Jüngeren

$\ddot{A}$ Translokation: $\mathrm{t}(15 ; 17) \rightarrow$

Retinoid-Rezeptor-Mutation

D Promyelozyten im Blut

Ko DIC, Fibrinolyse

T Vit.-A-Gabe uU kurativ! (triggert Blastenmaturierung)

\section{Akute lymphatische}

Leukämie (ALL)

Syn.: Akute Lymphoblasten-

Leukämie

$P$ ensteht aus lymphatischer (Prä-B/Prä-T) Precursor-Zelle (streng genommen nur, wenn primär in Km entstehend, dann $=\mathrm{B} / \mathrm{T}-\mathrm{ALL}$; wenn peripher dann = lymphoblastisches Lymphom, ggf. mit sekundärem Km-Befall) $\sim 80 \%$ B-ALL:

Kleinkinder, idR ex Km $\sim 20 \%$ T-ALL:

KiJu, idR ex Peripherie

D Immunphänotypisierung!

T multiple Cx über Jahre

Pr Genotyp ausschlaggebend, b. Ki: Remissionsrate $~ 90 \%$.

Aus lymphatischer Reihe
Histiozytäre u.

dendritische Zell-Neoplasien

E sehr selten

P ex Histiozyten (gewebsständige Makrophagen) oder Langerhans-Zellen (APCs der Haut); oft iF BRAF-Mutation

- Langerhans-Zell-Histiozytose: disseminiert 0 . limitiert auf 1 Organ, zB

- Knochen: Kinder $(<3 \mathrm{~J}, \mathrm{~m}>\mathrm{f})$ Pulmonal: Ältere (idR Raucher) - Haut: häufig ekzematös

- Histiozytäres Sarkom Ex reifen interstitiellen Histiozyten, lokal begrenzt (Haut, LK Darmtrakt)

T ZT BRAF-Inhibitoren

Spotlight: Hämatologische Neoplasien mit sekundärem Knochenmarks-Befall, oder: der Sinn einer anatomischen Einteilung

Ein grosser Teil der lymphatischen Neoplasien entsteht im Gegensatz zu den oben genannten myeloischen Neoplasien (MPN, MDS, AML etc.) primär ausserhalb des Km, idR in Lymphknoten. Ihre Vorläuferzellen entstammen jedoch im weitesten Sinne dem Km. Einige unter ihnen befallen im Krankheitsverlauf regelmässig das Km (= sekundärer, ,Ir̈̈ckwärtiger“ Befall), Beispiele sind:

$\rightarrow$ Chronische lymphatische Leüämie (Kap 19)

$\checkmark$ Haarzellleukämie (Kap. 19)

$\rightarrow$ Multiples Myelom (Kap. 19)

- Lymphoplasmozytisches Lymphom (Kap. 19) Diese didaktisch nützliche, anatomische Sichtweise ist historisch bedingt; sie trennt lymphatische Neoplasien mit Km- u./o. Blutbeteiligung (=Leukämie), von solchen, die als Masse vorliegen (Lymphom). Es wurde jedoch zusehends klar, dass jedes „Lymphom“ sich uU zu einem leukämischen Bild entwickeln kann u. jede „Leukämie“ gelegentlich mit einer Massenläsion auftreten kann. Zudem können neue Methoden nachweisen, dass für das Therapieansprechen entscheidend die genetische Ausstattung der Tumorzelle ist u. nicht der Ort. Neue Klassifikationen teilen daher weniger nach Lage, sondern nach Art der Tumor-Ursprungszelle ein (anhand Morphologie, Immunphänotyp u. Zytogenetik). IdF wurden einige bisher als unterschiedlich betrachtete Entitäten zu einer Diagnosegruppe zusammengefasst. (zB B-ALL u. prä-B-Zell-Lymphoblasten-Lymphom: beide enstehen aus Precursor B-Zelle, einmal im Km, einmal in Lymphknoten).

ABI Anämie, Blutung, Infekte (=Symptome b. Km-Befall)

APC Antigenpräsentierende Zelle

ASS Acetyl-Salicyl-Säure

BS Blastenschub

CEPBA CCAAT/enhancer binding protein alphe

CX Chemotherapie
DLBCL Diffuse large B-cell lymphoma

HSCT Hematopoetic stem cell transplantation (autolog o. allogen)

HU Hydroxyurea

ITP Idiopathische thrombozytopenische Purpura

IPSS-R Revised International Prognostic Scoring System

$\mathrm{Km}$ Knochenmark
LAP Alkalische Leukozytenphosphatase ${ }^{3}$

MDS Myelodysplastische(s) Syndrom(e)

MKZ Megakaryozyten

MPN Myeloproliferative Neoplasie(n)

NSD Nebenschilddrüse

$\mathrm{Rx} \quad$ Radiotherapie 
Thymus

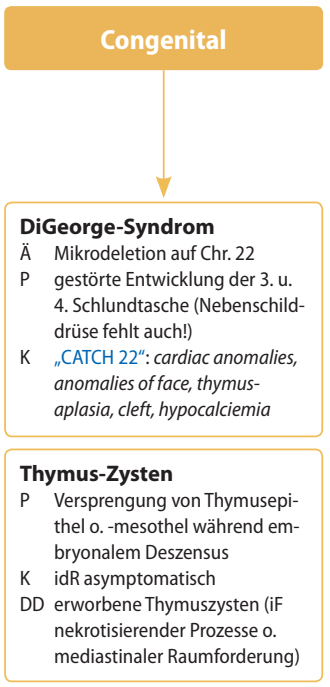

\section{Infektiös / Inflammat.}

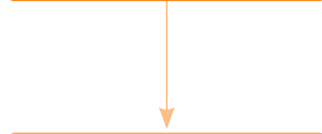

Lymphofollikuläre Thymitis

$\ddot{A}$ assoziiert mit Myasthenia gravis, SLE, Rheumatoider Arthritis, Morbus Basedow

Mi Ausbildung von B-Zell-Follikeln (gibt es sonst nicht in Thymus!)

\section{Stressinvolution}

Ä viraler o. bakterieller Infekt, Sepsis

Mi im Akutstadium zahlreiche Nekrosen u. Apoptosen

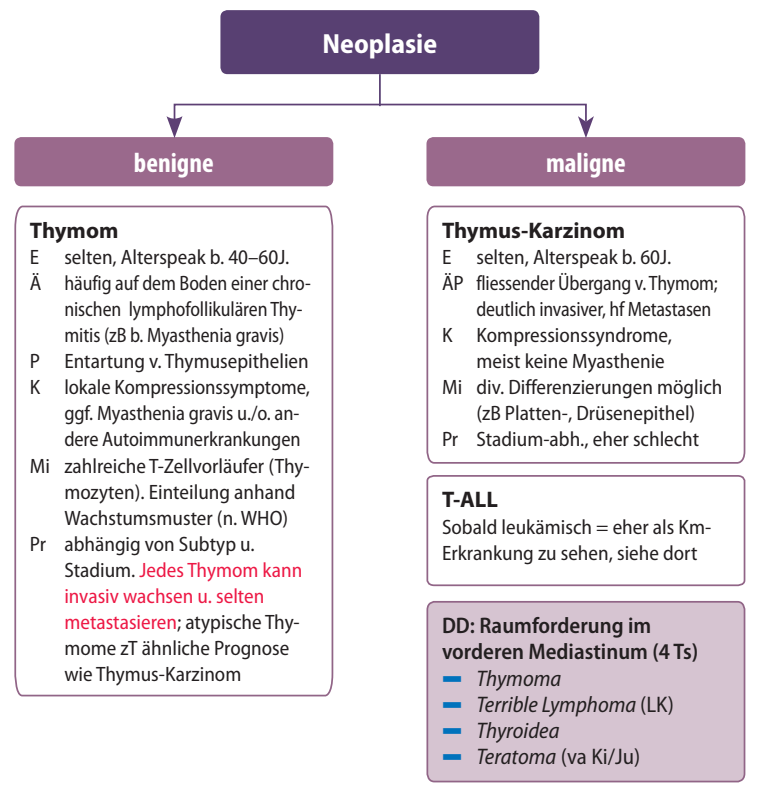

Fokus: angeborene Immundefekte

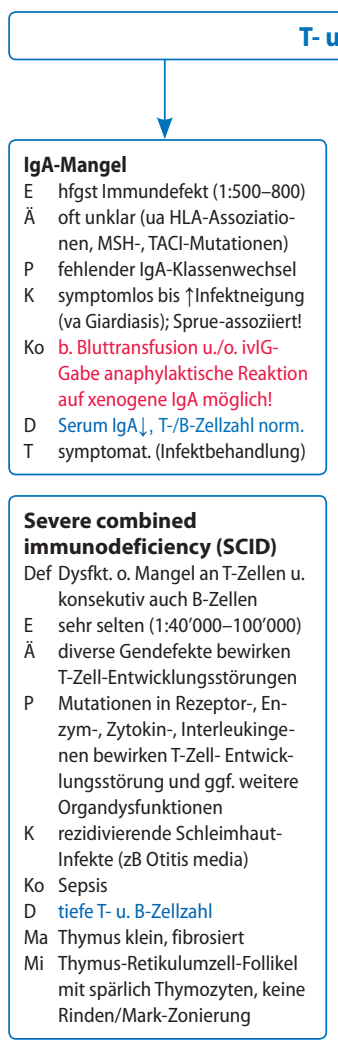

T- u. B-Zellen

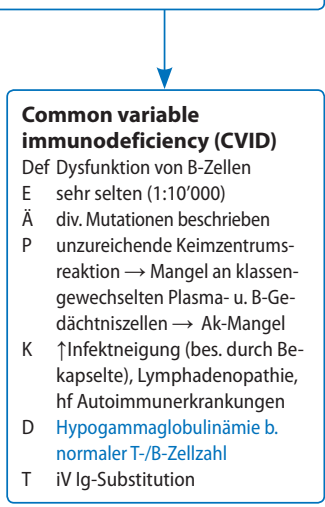

X-linked Agammaglobulinemia

Syn.: Bruton-Syndrom

Def fehlende B-Zellen

E sehr selten (1:250'000)

$\ddot{A} \quad$ X-gelinkte Mut. in B-Zell-Tyrosinkinase $\rightarrow \varnothing$ B-Zellentwicklung

K s. CVID

D Hypogammaglobulinämie mit tiefer B-Zellzahl, T-Zellzahl norm. T iV Ig-Substitution

Hyper-IgE-/lgM-Syndrom (Hiob)

$E$ extrem selten

Ä div. Mutationen

K $\uparrow$ Infektneigung mit zT "kalten“ Abszessen (øperifokale Rötung)

D IgE-, IgM-Serumspiegel $\uparrow$

T symptomat. (Infektbehandlung)
Phagozytose-Probleme

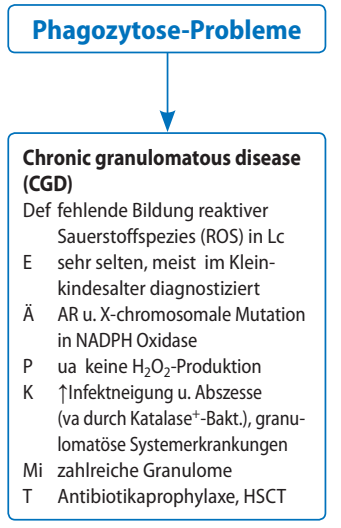

Chediak-Higashi-Syndrom

E extrem selten

$\ddot{A} \quad$ AR-Mutation in LYST-Gen

$P$ fehlerhafter Lysosomentransport (ua in Granulozyten, Melanozyten)

K pyogen-bakterielle Infekte $u$. partieller Albinismus

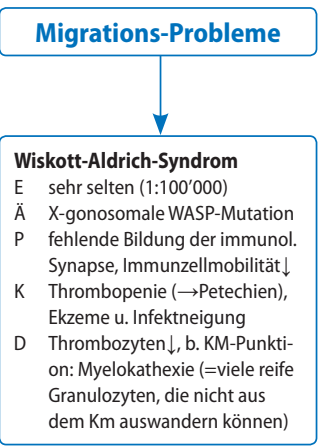

Leukozyten-Adhäsions-Defizit

E extrem selten

Ä AR-Mutation in Integrin-Rez.

P gestörte Leukozyten-Adhäsion u. -Diapedese

K ausbleibende Nabelschnurseparation, zahlreiche bakt. Infekte ab Neonatalperiode

D Diff.-Blutbild: (Lc stark erhöht), Flowzytometrie

T HSCT
1 Imitieren uU eine primäre Km-Neoplasie, wobei deren Ursache systemischer Natur o. nur das Km betreffend sein kann

2 Beachte: MPN, MDS U. AML bilden ein Spektrum von myeloischen Neoplasien: ausgehend von erhöhtem ProgenitorzellUmsatz ist Ausreifung genügend (MPN), schlecht (MDS) o. øvorhanden (AML)
${ }^{3}$ Die LAP ist im normalen reifen Granulozyten vorhanden. Das hilft b. der Abgrenzung CML $\leftrightarrow$ leukämoide Reaktion (sonstiger starker Lc-Anstieg): Lc++, nGZ++, LAP++

${ }^{4} \mathrm{ALL}=$ Überbegriff für $>20 \%$ maligne lymphat. Zellen in $\mathrm{KM}$ o. Blut. Ursprung kann Km-Zelle sein ( $\rightarrow$ Lymphoblastische Leukämie) o. entartete periph. B-/T-Zellen ( $\rightarrow$ Lymphoblastisches Lymphom) 


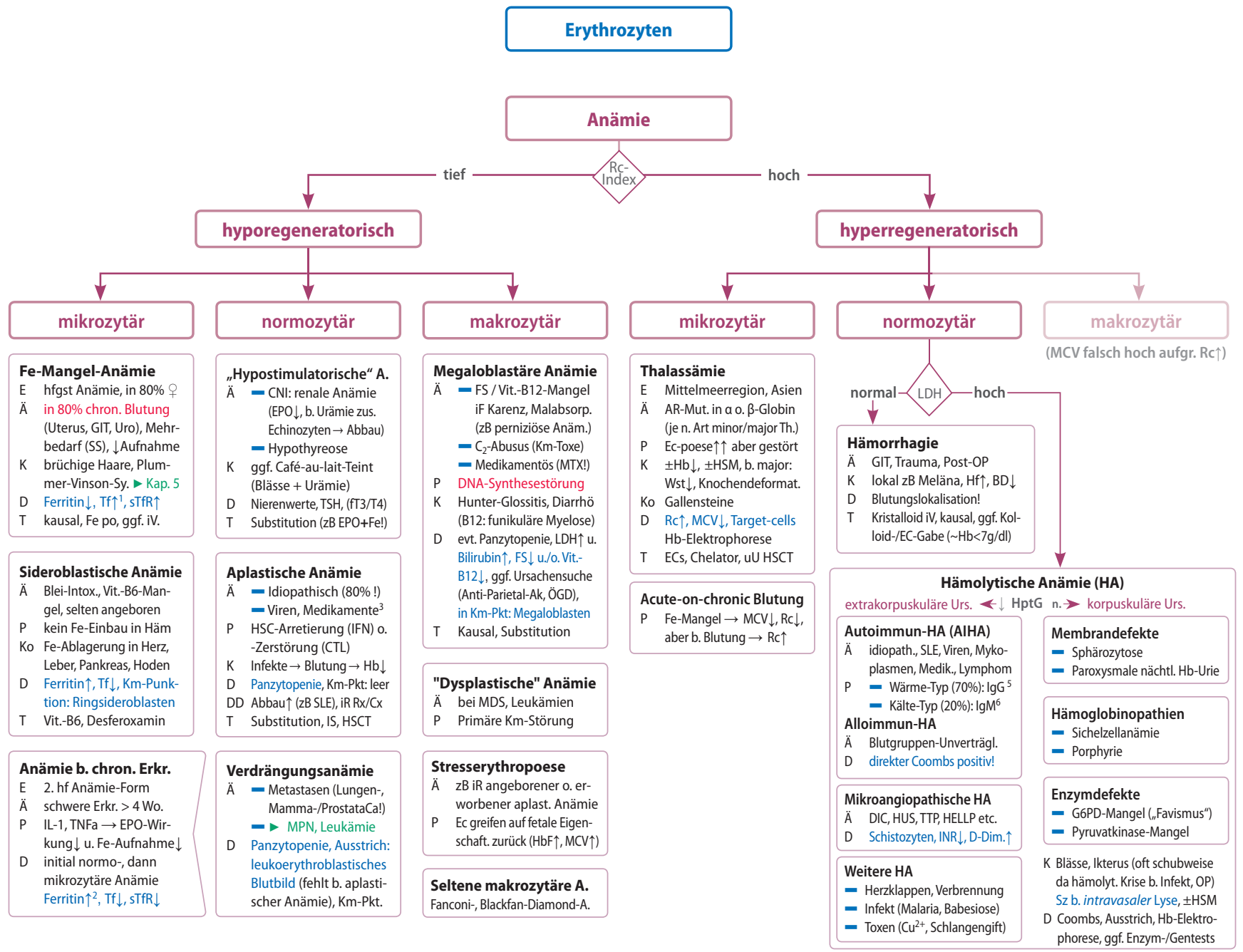

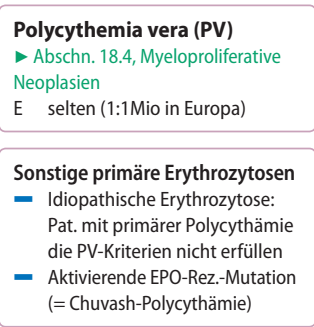

AlHA Autoimmunhämolytische Anämie

$\mathrm{COHb}$ Carboxyhämoglobin

CTL Cytotoxische T-Lymphozyten (CD8 $\left.{ }^{+}\right)$

EPO Erythropoietin

EC Erythrozyten-Konzentrat

$\mathrm{Fe}$ Eisen

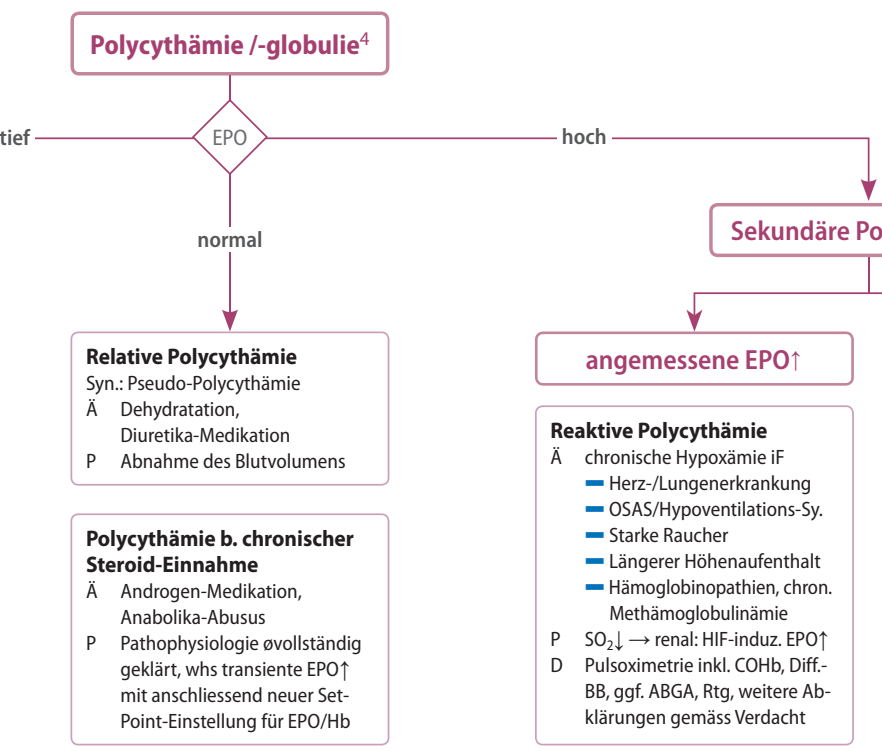

HSC(T) Hämatopoetische Stammzell(en) (Transplantation

HSM Hepatosplenomegalie

HUS Hämolytisch-urämisches Syndrom

IFN Interferon

MTX Methotrexat

IS Immunsuppression
G6PD Glucose-6-Phosphat-Dehydrogenase

$\mathrm{Hb}$ Hämoglobin

HELLP Hemolysis, elevated liver enzymes, low platelets

HIF Hypoxia-inducible Factor

HptG Haptoglobin

\section{unangemessene EPO}

Paraneoplastische

Polycythämie

Ä ua b. Nierenzell-CA, Phäochromocytom, HCC ( Kap. 8), cerebelläres Hämangioblastom

P paraneoplastische, autonome EPO-Sekretion

D U-Status (Ec?), US-Abdomen

Nierenarterienstenose

Kap. 10, Niere

renale Hypoxämie $\rightarrow$ HIF-induz. EPO $\uparrow$ 


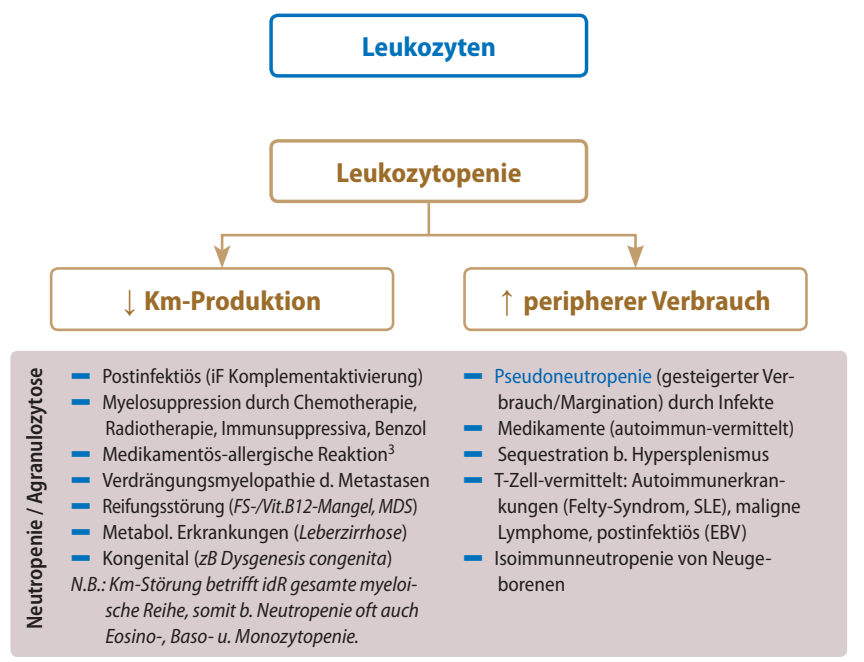

Eosinopenie, Basopenie, Monozytopenie: Schwer zu quantifizieren, da physiologisch zT sehr tiefe Zellzahl. IdR „Begleitpenien“ b. Agranulozytose (siehe oben). Isolierte Eosino- $u$. Basopenie zB bei Stress

(Trauma, Schock), Cushing-Syndrom od. ZB Typhus abdominalis. Seltene hereditäre Ursachen beschrieben.

- Postinfektiös
- Medikamente (Immunsuppressiva, CTC)
- Viren (Masern, HIV, va T-Lymphozytopenie)
- Autoimmunerkrankungen (SLE)
- Myelosuppression durch Chemotherapie
o. Radiotherapie
- Sehr selten: hereditär

- Cushing-Syndrom, CTC, Stress

(1)

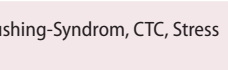

